\title{
An overview of the information distribution system at the Advanced Photon Source
}

\author{
M. Ramanathan, M. Smith, N. Arnold, F. Lenkszus, R. Laird, K. Evans, Jr., J. Anderson, \\ and K. Sidorowicz \\ Advanced Photon Source, Argonne National Laboratory, 9700 South Cass Avenue, Argonne, Illinois 60439
}

(Presented on 22 August 2001)

\begin{abstract}
The Advanced Photon Source (APS) has been in operational mode for more than 5 yr. Currently there are over 40 beamlines in various phases of operation. The control system of choice at the APS is the Experimental Physics and Industrial Control System (EPICS). We have provided various interfaces to the beamlines from the APS control system. An overview of the various systems will be discussed. The General Control System Information (GCSI) uses dedicated computers as EPICS process variable gateways to provide data from the APS control system to each beamline. The GCSI architecture makes the APS control system secure, yet has the flexibility of providing access control to any data available on the APS control system. In addition, the gateway reduces the load on the APS control system equipment by making only one connection for each process variable accessed by multiple users. Each sector, consisting of a bending magnet and insertion device beamlines, is provided its own gateway, which resides on the local sector network. This scheme has the advantage of providing network security and more reliable operation. To provide real-time accelerator data to beamlines, each sector has been provided a chassis to display the storage ring current and other relevant information. These data are transmitted via a direct fiber link from the APS control system hardware to the beamlines. The beamlines are also provided VME-based hardware and associated EPICS software to retrieve key information provided via this fiber link. Some of the information on this link is beam current, lifetime, injection status, and sector specific information such as shutter status, insertion device gap, and energy, and storage ring and front-end beam position monitor signals. The data rates on this link are typically $10 \mathrm{~Hz}$ but can be as high as $272 \mathrm{kHz}$. This scheme allows the beamlines using EPICS-based software to seamlessly use the data from the APS control system without excessively impacting the system. For high-precision x-ray timing experiments, a new scheme has been designed to distribute the radio-frequency timing signals to the beamlines. Using VME-based hardware and EPICS software, the bunch clock signal is generated at the beamline for use in timing experiments. Data are provided at the radio frequency of $352 \mathrm{MHz}$. Beamlines have also been provided with hardware and software to generate the bunch clock signal for timing experiments. (C) 2002 American Institute of Physics. [DOI: 10.1063/1.1436544]
\end{abstract}

\section{INTRODUCTION}

Prior to the commissioning of the beamlines at the Advanced Photon Source (APS), the need arose for the APS control system to communicate with these beamlines. ${ }^{1}$ The earliest implementation was to provide each beamline with a special receiver to display the beam current. As time passed the system was expanded to incorporate additional functionality. At the heart of the APS are the insertion devices (IDs), the source of $x$ rays for all the ID beamlines. The need arose for the beamlines to be able to control the ID. Owing to network security and the need to reduce impact on performance of the controls network, the control of these devices had to be routed through a gateway. The time structure of the $\mathrm{x}$-ray beam produced by the APS is very useful for certain experiments. The beamline synchronizes their experimental detectors with the arrival of the $\mathrm{x}$-ray beams. This required the APS to provide the beamlines with a timing signal to precisely synchronize their detectors. The implementation of all these systems will be discussed.

\section{DESCRIPTION}

At the APS the beamlines are configured as sectors, with each sector consisting of a bending magnet (BM) beamline and an ID beamline. ${ }^{1}$ Collaborative access teams in turn operate each sector. Each sector has its own network configuration, completely isolated with a router. Network firewalls are in the process of being installed. The overall information distribution at the APS can be classified into three categories based on their data rates and their functionality. The general control system information (GCSI) is based on Experimental Physics and Industrial Control System process variable (EPICS PV) gateways. ${ }^{2}$ GCSI can provide any data available on the APS control system to all sector beamlines. The real-time accelerator data (RAD) are provided via direct link to the sectors from the APS control system. This system provides a limited set of data from the APS control system at fairly high data rates. The third system is the high-precision x-ray timing (HPXT) signal provided to the sectors via dedicated fiber optics link to the sectors. The HPXT signal is used for very 


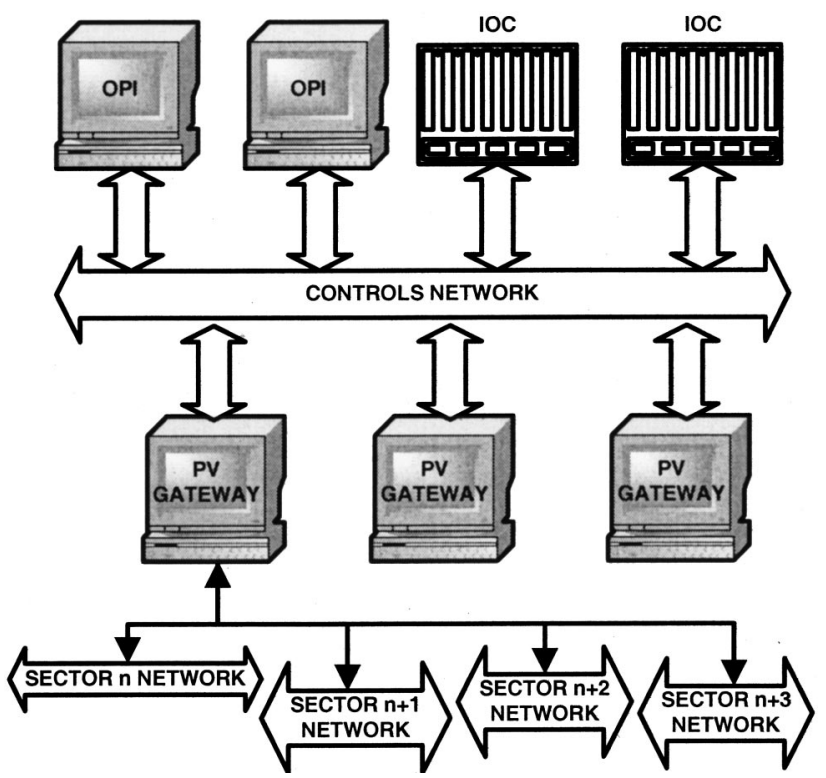

FIG. 1. Network topography for the GCSI scheme.

specific experiments involving synchronization between the experimental detectors and the arrival of the x-ray beam at the detector.

\section{A. General control system information}

The EPICS-based APS control system uses the distributed computing approach with an Ethernet network forming the backbone. In this system hardware devices are controlled by either VXI or VME crates acting as input/output controllers (IOCs). The processors in these VME/VXI crates are attached to the network. The control system network has two Sun Microsystems file servers. User interface to the control system is via UNIX- or PC-based computers. The control system network consists of 10/100 Ethernet links to the IOC and the file servers. To provide EPICS process variables from theses IOCs around the ring to the beamlines, dedicated Sun Microsystems Ultra 10 computers are used. Figure 1 shows the network topography for the GCSI scheme at the APS. ${ }^{1}$ Each of the Ultra 10 computers acting as a PV gateway has one primary fast Ethernet connection to the APS controls network. In addition, each PV gateway has a secondary quad fast Ethernet controller. Each of the PV gateways serves four sectors. The secondary Ethernet ports are in turn directly connected to the sector subnet directly. Using media converters and fiber optic cables, the connections to each sector's subnet is accomplished from the central computer locations. At the current time there are six dedicated PV gateways with one additional hot spare operating. All these machines are identical in configuration. The PV gateways are operating under the Solaris 5.8 operating system. These computers are completely stand alone. Each of these PV gateways acts as the domain name server as well as timeservers for the sector-specific networks. EPICS PV gateway software was developed ${ }^{3}$ to provide access to the EPICS PV from the controls network to the user transparently, while at the same time providing the required network isolation. The PV gateway software is bidirectional. It can honor requests for control and at the same time provide monitoring

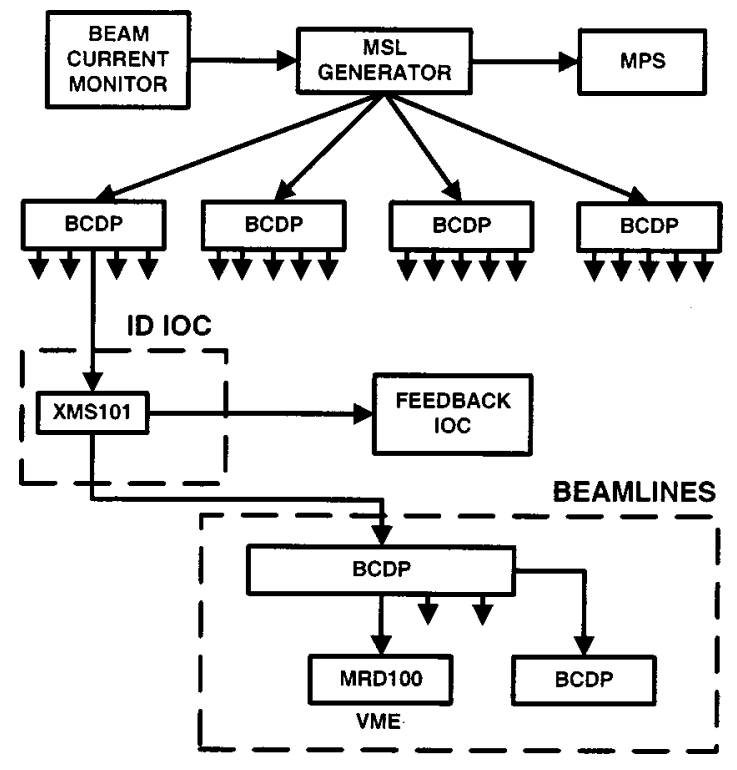

FIG. 2. Layout of the RAD distribution system.

capability for the beamlines. The PV gateway has rule sets that can be configured to allow/deny access to any PV. The PV gateway also has logging capability. At the current time the data rate through the PV gateway is limited to about 10 $\mathrm{Hz}$.

\section{B. Real-time accelerator data distribution}

During commissioning of the storage ring a need arose for access to the stored beam current signal by many subsystems like the Orbit Correction System, the Machine Protection System, and also the experimental beamlines. The RAD distribution also known as Machine Status Link $(\mathrm{MSL})^{4}$ was initially designed to provide a digitized beam current signal, injection status, and also the revolution clock signal $(P 0)$ via a single fiber optic link. The beam current signal along with the injection signal and a few extra bits of data are packed into a word and are transmitted serially at the $P 0$ rate, which is approximately $270 \mathrm{kHz}$. A beam current distribution panel (BCDP) was designed to receive the fiber optic signal and decode the information and provide the data in various formats, including a light emitting diode current display module. The BCDP also has fan-out capability to distribute the signal via fiber optic to other BCDP modules.

Figure 2 is a layout of the RAD distribution system. The machine status word (MSW) is generated at one central location and is distributed to all other systems. Main hubs are located at four places around the storage ring. Each of these locations has a BCDP module with the capability to fan out the signal to ten locations each. The MSW is transmitted from these modules to each ID IOC. A VME module (XMS 100) was designed to accept the MSW signal, append additional words, and transmit the expanded data to the beamline for appropriate decoding. The data are transmitted at the $P 0$ clock rate. The XMS 100 was originally designed to accept data from $\mathrm{BM}$ front-end $\mathrm{X}$-ray beam position monitors (XBPMs) and the ID front-end XBPM. In addition, at the ID IOC additional data (e.g., ID gaps, shutter status, etc.) can also be added to the list of data to be transmitted to the 
beamlines. Recently, owing to changes in the configuration of the XBPM, the XMS module was modified. The new XMS 101 has one output for the beamlines, which is transmitted at the $P 0$ rate, and an additional output for the storage ring feedback system to provide the ID gaps. The signal is transmitted to each sector via a single fiber. Each sector is provided with one BCDP module and one VME (MRD100) module. The BCDP has the provision to fan out to more of the units at the beamline. Software is provided to the beamlines $^{5}$ to decode all the signals in their IOC. Even though the data are transmitted at about $270 \mathrm{kHz}$, owing to the fact that there are about 26 words of data transmitted, the final data rate is about $10 \mathrm{kHz}$. Both the BCDP and the MRD100 modules have a $P 0$ output. The $P 0$ signal occurs each time the particle beam makes one turn around the storage ring. These $P 0$ signals can be used for some timing experiments.

\section{High precision x-ray timing distribution}

Recent advancements in detector technologies and the need to perform experiments in the time domain have increased the requirements for precise timing between the arrival of the $\mathrm{x}$-ray photon beam at the experiment and the detector. Performing these time-dependent experiments requires a timing signal synchronized with the arrival of the $\mathrm{x}$-ray beam at the experiment.

A Bunch Clock Generator was designed ${ }^{6}$ to meet the needs of the timing experiments. The APS storage ring operates the rf at $352 \mathrm{MHz}$. There are 1296 discrete possible locations for the particle beam to be stored. A VME Bunch Clock Generator module (BCG100) was designed to provide pulses for any of the 1296 bucket locations at the $P 0$ rate. The BCG100 accepts a low-level rf $352 \mathrm{MHz}$ signal and the $P 0$ signal to generate the pulses. The pulses are NIM-level outputs and are about $1 \mathrm{~ns}$ wide. Coarse and fine programmable delays are provided to adjust the timing of the output to synchronize with the arrival of the x-ray beam at the experimental stations. Software loaded in the beamline ${ }^{7}$ IOC where the BCG100 resides retrieves the storage ring fill pattern from the PV gateway and uses the data to generate the corresponding pulses for all the buckets that have particle beam loaded in it. For specific experiments if the user desires, they can load their own pattern into the BCG100 memory for generation of a specific pulse train.

Figure 3 is a layout of the HPXT distribution system. The low-level rf $352 \mathrm{MHz}$ signal can be transmitted using dedicated single-mode fiber from one central location, but a more cost-effective method was employed. A scheme was designed to divide the rf signal by 8 and distribute the resultant $44 \mathrm{MHz}$ via multimode fiber and subsequently regenerate the signal by employing a phase-locked loop circuit to multiply by 8 . This design is very cost effective, since the multimode fiber was already in existence at the various locations and due to the low cost of multimode transmitters and receivers. The $44 \mathrm{MHz}$ rf/8 signal along with the $270 \mathrm{kHz} P 0$ is distributed around the storage ring to all the odd sector beam position monitor (BPM) racks. Each BPM rack fans

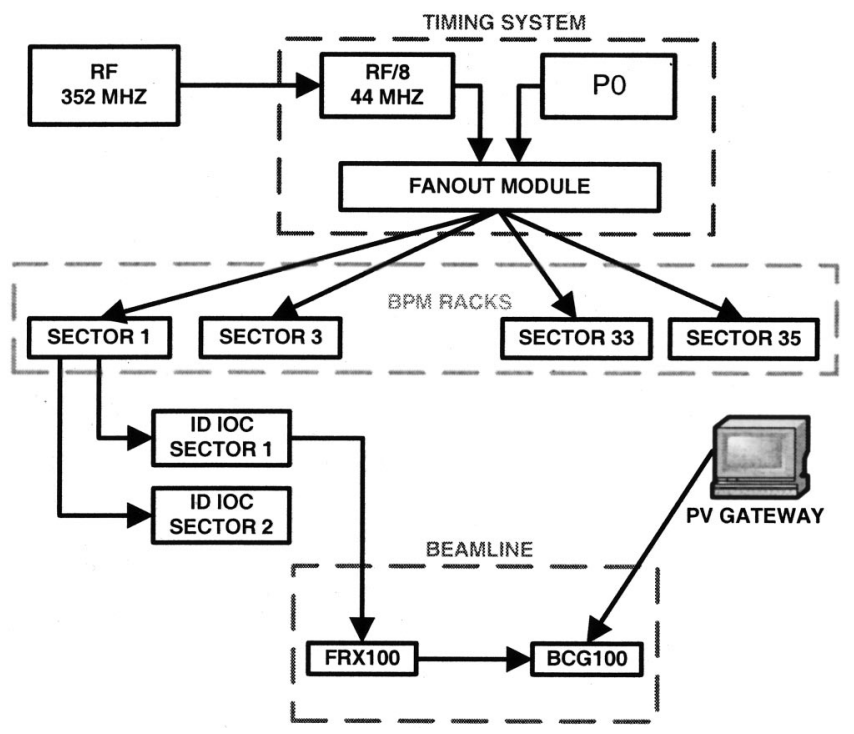

FIG. 3. Layout of the HPXT distribution system.

out the signals to two sectors. The signals are then transmitted to the ID IOC racks. At the ID IOC rack the $P 0$ signal, $44 \mathrm{MHz}$ rf/8 signal, and the MSL signal are all packed into a six-conductor fiber bundle (three spare fibers) and transmitted to the beamlines. At the beamline a VME module (FRX100) is used to convert the fiber signals to copper and uses a phase-locked loop to regenerate the $352 \mathrm{MHz}$. The NIM level $352 \mathrm{MHz}$ output is used as a clock input to the BCG100 module to generate the bunch clock signals.

\section{CONCLUSION}

Presently there are 25 sectors out of a possible 35 operating at the APS. All the beamlines use a PV gateway for access to the APS control system. The BCDP has been provided to each sector and is being fully utilized. The bunch clock signal is being used by some beamlines at this time. More and more of the users are starting to make use of the time structure of the beam, and hence the need for the bunch clock signals is increasing. To increase reliability, we are designing a bidirectional communication link between the sectors and the ID control system by an alternative method to the PV gateway.

\section{ACKNOWLEDGMENTS}

The authors wish to thank everyone at APS who contributed to this project in different ways. This work is supported by the U.S. Department of Energy, Office of Basic Energy Sciences under Contract No. W-31-109-ENG-38.

${ }^{1}$ D. E. Moncton and G. K. Shenoy, Nucl. Instrum. Methods Phys. Res. A 266, 1 (1988).

${ }^{2}$ EPICS, http://www.aps.anl.gov/epics

${ }^{3}$ J. Kowalkowski, J. Anderson, and K. Evans Jr. (private communications).

${ }^{4}$ http://www.aps.anl.gov/asd/controls/hardware/ beamStatusLink/beamStatusLink.fm.html

${ }^{5}$ http://www.aps.anl.gov/aod/blops/MSL/MSL.html

${ }^{6}$ F. Lenkszus and R. Laird, 1997 Particle Accelerator Conference (IEEE, New York, 1998), Vol. 2, pp. 2490-2492.

${ }^{7}$ http://www.aps.anl.gov/aod/blops/BCLOCK/BCLOCK.html 